\title{
POST DURAL PUNCTURE HEADACHE
}

Prabhavathi R ${ }^{1}$, Chaitanya Kumar G², Narasimha Reddy $\mathrm{P}^{3}$

\section{HOW TO CITE THIS ARTICLE:}

Prabhavathi R, Chaitanya Kumar G, Narasimha Reddy P. "Post Dural Puncture Headache". Journal of Evolution of Medical and Dental Sciences 2014; Vol. 3, Issue 36, August 18; Page: 9426-9434,

DOI: $10.14260 /$ jemds/2014/3216

ABSTRACT: Headache is a frequent complication of dural puncture, particularly with a large-bore needle. Clinical and laboratory research over the last 30 years has shown that use of small-gauge needles, particularly of the pencil-point design, is associated with a lower risk of PDPH Epidural blood patching is a well-accepted therapeutic modality for dural puncture headache; it seemed reasonable that the sooner done, the shooter would be the duration of the headache. We had noticed an unusually high failure rate of epidural blood patching done shortly after dural puncture.

KEYWORDS: Post dural puncture headache, Cerebrospinal fluid, large gauge needle, Epidural blood patch.

INTRODUCTION: A dural puncture with an epidural needle is a stressful event of the anesthesiologist and the patient, especially when it occurs in the parturient. Typical concerns are raised: Will the patient develop a headache? Is there anything that can be done to prevent it? How should a postdural puncture headache (PDPHA) be treated? If she gets a headache it will become my headache? Headache remains a major problem to the obstetric anesthesiologist.

HISTORY: The history of spinal anesthesia can be traced back to the late 1800s when Wynter and Quincke aspirated cerebrospinal from patients with tuberculous meningitis in an attempt to lower intracranial pressure.[1] Shortly thereafter, John Corning attempted to use spinal cocaine injection for the treatment of habitual masturbation. Whether it cured the ailment it was intended for being unknown. August Bier performed spinals on himself and eight other subjects using 10-15 mg of cocaine. Four of the nine people, including Professor Bier, developed PDPH. ${ }^{[2]}$

\section{PATHOPHYSIOLOGY OF DURAL PUNCTURE:}

Anatomy of the spinal Dura Mater: The spinal dura mater is a tube extending from the foramen magnum to the second segment of the sacrum. It contains the spinal cord and nerve roots that pierce it. The dura mater is dense, connective tissue layer made up of collagen and elastic fibers. The classical description of the spinal dura mater is of collagen fibers running in a longitudinal direction. ${ }^{3]}$

Clinical teaching based upon this view of the dura recommends that a cutting spinal needle be oriented parallel rather than at right angles to these longitudinal dural fibers.[4,5] Orientating the needle at right angles to the parallel fibers, it was said would cut more fibers. The cut dural fibres, previously under tension, would then tend to retract and increase the longitudinal dimensions of the dural perforation, increasing the likelihood of a post spinal headache.

Clinical studies had confirmed that post dural puncture headache was more likely when the cutting spinal needle was oriented perpendicular to the direction of the dural fibres. However, recent light and electron microscopic studies of a human dura mater have contested this classical 
description of the anatomy of the dura mater. These studies describe the dura mater as consisting of collagen fibers arranged in several layers parallel to the surface.

Each layer or lamellae consist of both collagen and elastic fibres that do not demonstrate specific orientation.[6] The outer or epidural surface may indeed have dural fibres arranged in a longitudinal direction, but this pattern is not repeated through successive dural layers.

Recent measurements of dural thickness have also demonstrated that the posterior dura varies in thickness, and that the thickness of the dura at a particular spinal level is not predictable within an individual or between individuals. Dural perforation in a thick area of dura may be less likely to lead to a CSF leak than a perforation in a thin area, and may explain the unpredictable consequences of a dural perforation.

Cerebrospinal Fluid: CSF production occurs mainly in the choroid plexus, but there is some evidence of extrachoroidal production. About $500 \mathrm{ml}$ of CSF is produced daily $(0.35 \mathrm{ml} / \mathrm{min}) .{ }^{[7]}$ The CSF volume in the adult is approximately $150 \mathrm{ml}$, of which half is within the cranial cavity. The CSF pressure in the lumbar region in the horizontal position is between 5 and $15 \mathrm{~cm} \mathrm{H}_{2} \mathrm{O}$. The pressure of the CSF in children rises with age, and may be little more than a few $\mathrm{cm} \mathrm{H}_{2} \mathrm{O}$ in early life.

Duramater and Response to Trauma: The consequences of perforation of the spinal or cranial dura are that there will be leakage of CSF. Neurosurgical experience of dural perforation is that even minor perforations need to be closed, either directly or through the application of synthetic or biological dural graft material. Failure to close the dural perforation may lead to adhesions, continuing CSF leak, and the risk of infection.

Experimental studies show that deliberate dural defects in the cranial dura of dogs took approximately one week to close. The closure was facilitated through fibroblastic proliferation from surrounding tissue and blood clot.

It is therefore possible that a spinal needle carefully placed in the subarachnoid space does not promote dural healing, as trauma to adjacent tissue is minimal. Indeed, the observation that blood promotes dural healing agrees with Gormley's original observation that bloody taps were less likely to lead to a post - dural puncture headache as a consequence of a persistent CSF leak.

Needle tip deformation and Dural Perforation: It has been proposed that contact with bone during insertion may lead to spinal needle tip deformation. Damaged needle tips could lead to an increase in the size of the subsequent dural perforation. Recent in vivo studies have demonstrated that the cutting type spinal needle is more likely to be deformed after bony contact than comparable sized pencil - point needles.

Consequences of Dural Puncture: Puncture of the dura has the potential to allow the development of excessive leakage of CSF. There will be negative pressure in the epidural space and hence CSF leaks from a higher pressure area to a space with lower pressure, Excess loss of CSF leads to a demonstrable reduction in CSF Volume. After the development of post dural puncture headache, the presence of a CSF leak has been confirmed with radionuclide cisternography, radionuclide myelograph, manometric studies, epiduroscopy. 
The adult subarachnoid pressure of $5-15 \mathrm{~cm} \mathrm{H}_{2} \mathrm{O}$ is reduced to $4.0 \mathrm{~cm} \mathrm{H}_{2} \mathrm{O}$ or less. The rate of CSF loss through the dural perforation is generally greater than the rate of CSF production $(0.35 \mathrm{ml} / \mathrm{min})$, Particularly with needle sizes larger than $25 \mathrm{G}$. Although the loss of CSF and lowering of CSF pressure is not disputed, the actual mechanism producing the headache is unclear. There are two possible explanations. First, the lowering of CSF pressure causes traction on the intracranial structures in the upright position.

These structures are pain sensitive, leading to the characteristic headache.[8] Secondly, the loss of CSF produces a compensatory venodilatation (Monro - Kellie doctrine). The Monro - Kellie doctrine, or hypothesis, states that the sum of volumes of the brain, CSF, and intracranial blood is constant. The consequence a decrease in CSF volume is a compensatory increase in blood volume. The venodilation is then responsible for the headache. ${ }^{(9)}$

Needle Quality: Reducing the size of the spinal needle has made a significant impact on the incidence of post - spinal headache. The incidence is - $40 \%$ with a $22 \mathrm{G}$ needle; $25 \%$ with a $25 \mathrm{G}$ needle; $2 \%$ $12 \%$ with a 26G Quincke needle; and $<2 \%$ with a $29 \mathrm{G}$ needle. However, technical difficulties leading to failure of the spinal anesthetic are common with needles of $29 \mathrm{G}$ or smaller. Needle modifications since that time, such as the Sprotte and Atraucan needles, promise further reductions in post spinal headache.

Needle Size: Large spinal needles will clearly produce large dural perforations where the likelihood of a dural puncture headache is high. Conversely, the smaller needles produce small dural perforations with a lower incidence of headache. Fine gauge spinal needles, 29G or smaller, are technically more difficult to use, and for spinal anesthesia at least, are associated with a high failure rate. A balance has to be struck between the risks of dural puncture headache and technical failure. 25G, 26G and 27G needles probably represent the optimum needle size for spinal anesthesia.

Needle Orientation: There are many clinical, and laboratory, studies that lend credence to the hypothesis that the perpendicular orientation of the bevel of a spinal or epidural needle leads to a reduction in the incidence of post - dural puncture headache.

Operator skill level and Fatigue: It has been suggested that the incidence of inadvertent dural puncture during epidural anesthesia is inversely related to operator experience. However, sleep deprivation, operation fatigue and the effect of night work may be a confounding variable producing the higher incidence of inadvertent dural puncture in junior personnel performing epidural analgesia.

Obstetrics: The parturient is at particular risk of dural puncture and the subsequent headache because of their sex,(10) young age, and the widespread application of epidural anesthesia. In parturients receiving epidural anesthesia the incidence of dural puncture is between 0 and $2.6 \%$. The incidence is inversely related to the experience of the anesthesiologist, and is said to be reduced by orientation of the needle bevel parallel to the dural fibre.(11)

Loss of resistance to air confers a higher risk of dural puncture than loss of resistance to fluid. After a dural puncture with a 16G Tuohy needle, up to $70 \%$ of subjects will report symptoms related to low CSF pressure. Despite the high incidence of headache consequent upon dural puncture with a 
Tuohy needle, the anesthesiologist needs to consider a differential diagnosis, as intracranial hematoma, or tumor presenting with similar symptoms to, or in association with, a post - dural puncture headache has been described.

Children: Post dural puncture headache is reported as uncommon in children.(12,13)

Symptoms: The International Headache Society has defined a PDPH as a bilateral headache that develops within 7 days after lumbar puncture and disappears within 14 days after the lumbar puncture. The headache worsens within 15 minutes of assuming the upright position and disappears or improves within 30 minutes of resuming the recumbent position.(14) These symptoms are helpful in distinguishing from migraine headaches.

It usually occurs in the frontal, occipital, or both areas, but also may involve the neck and upper shoulders. Although it generally occurs within 48 hours of the dural puncture, it can occur later than 3 days in $25 \%$ of the cases. ${ }^{(15)}$

Other symptoms accompanying PDPH include nausea, vomiting, visual disturbances, and hearing alteration. Visual disturbances are due to dysfunction of the extraocular muscles from transient paralysis of the motor nerves of the eyes (CN III, IV and IV).(16) CN IV is the most frequently affected because of its long intracranial course and can be corrected with epidural blood patch.(17)

Diagnosis: The history of accidental or deliberate dural puncture and symptoms of a postural headache, neck ache and the presence of neurological signs, usually guide the diagnosis. A diagnostic lumbar puncture may demonstrate a low CSF opening pressure or a 'dry tap', a slightly raised CSF protein, and a rise in CSF lymphocyte count. An MRI may demonstrate: diffuse dural enhancement, with evidence of a sagging brain; descent of the brain, optic chiasm, and brain stem; obliteration of the basilar cisterns; and enlargement of the pituitary gland. CT myelography, retrograde radionuclide myelography, cisternography, or thin section MRI can be used to locate the spinal sources of the CSF leak.

Differential Diagnosis: The diagnosis of post - dural puncture headache is frequently clear from the history of dural puncture and the presence of a severe postural headache. However, it is important to consider alternative diagnosis as serious intracranial pathology may masquerade as a post dural puncture headache.

Diagnoses that may masquerade as post dural puncture headache include intracranial tumors, intracranial hematoma, pituitary apoplexy, cerebral venous thrombosis, migraine, chemical or infective meningitis, and nonspecific headache $(18,19,20)$. It has been estimated that $39 \%$ of parturients report symptoms of a headache unrelated to dural puncture following delivery. The largest follow up of post dural puncture headache is still that of Vandam and Dripps in 1956. They reported that $72 \%$ of headaches resolved within 7 days, and $87 \%$ had resolved in 6 months.

Prevention: Several maneuvers have been advocated to prevent a PDPH. Many recommend bed rest to prevent a PDPH. The first systematic examination of recumbency following dural puncture with a 22-gauge Quincke needle showed no difference if the patient rested 4 hours (11.6\%) and 24 hours 
(11.9\%).(21) Another study using 25-and 26-gauge Quincke needles found a higher incidence (55\% Vs. $25 \%)$ if the patient rested for 24 hours.(22)

\section{TREATMENT:}

Over View: Studies observing the effects of treatments in post dural puncture headache often fail to recognize that, with no treatment, over $85 \%$ of postdural puncture headaches will resolve within 6 weeks.

Psychological: It is important to give the patient a through explanation of the reason for the headache, the expected time course, and the therapeutic options available.

Simple: Bed rest has been shown to be of no benefit. Supportive therapy such as rehydration, acetaminophen, non-steroidal anti - inflammatory drugs, opioids, and anti-emetics may control the symptoms and so reduce the need for more aggressive therapy, but do not provide complete relief.

Posture: If a patient develops a headache, they should be encouraged to lie in a comfortable position. The prone position raises the intra-abdominal pressure, which is transmitted to the epidural space and may alleviate the headache.

Abdominal binder: A tight abdominal binder raises the intra-abdominal pressure and it is transmitted to the epidural space and may relieve the headache. Unfortunately, tight binders are uncomfortable and are seldom used in current practice.

Caffeine: Caffeine is a central nervous system stimulant that amongst other properties produces cerebral vasoconstriction. It is a available in an oral and i.v. form. The most frequently quoted work on the treatment of post dural puncture headache with caffeine is that of Sechzer. He evaluated the effects of one or two $0.5 \mathrm{~g}$ doses of i.v. Caffeine on subjects with established post dural puncture headache. There are some statistical and methodological flaws in this study, but it was concluded that i.v. Caffeine is an effective therapy for post dural puncture headache.

Dose: The dose now recommended for the treatment of post dural puncture headache is $300-500$ mg of oral or i.v. Caffeine once or twice daily (23).

Mode of Action: it is assumed that caffeine acts through vasoconstriction of dilated cerebral vessels. If cerebral vasodilatation were the source of the pain, cerebral vasoconstriction might limit the pain experienced.

Sumatriptan: The treatment for migraine headaches has focused on modification of cerebral vascular tone. Sumtriptan is a $5-\mathrm{HT}_{1 \mathrm{D}}$ receptor agonist that promotes cerebral vasoconstriction, in a similar way to caffeine. Sumatriptan (24) is advocated for the management of migraine and recently for post dural puncture headache. 


\section{REVIEW ARTICLE}

\section{EPIDURAL BLOOD PATCH (EBP):}

History: After the observation that bloody taps were associated with a reduced headache rate, the concept of the epidural blood patch has developed. The theory is that the blood, once introduced into the epidural space, will clot and occlude the perforation, preventing further CSF leaks. The high success rate and lower incidence of complications have established the epidural blood patch as the standard against which to evaluate alternative methods to treat post dural puncture headache.

Technique: With the patient in the lateral position, the epidural space is located with a Tuohy needle at the level of the supposed dural puncture or an intervertebral space lower. The operator should be prepared for the presence of CSF within the epidural space. Up to $30 \mathrm{ml}$ of blood is then taken from the patient's arm and injecting slowly through the Tuohy needle. Should the patient describe lancinating pain of dermatomal origin the procedure must be stopped.

There is no consensus as to the precise volume of blood required. Most practitioners now recognize that the 2 to $3 \mathrm{ml}$ of blood originally described by Gormley. (25) Is inadequate, and that 20$30 \mathrm{ml}$ of blood is more likely to guarantee success. Larger volumes, upto $60 \mathrm{ml}$, have been used successfully in cases of spontaneous intracranial hypotension. At the conclusion of the procedure, the patient is asked to lie still for one or, preferably, $2 \mathrm{~h}$, and is then allowed to walk.

Contraindications include those that normally apply to epidurals, but include a raised white cell count, pyrexia and technical difficulties. Limited experience with HIV - positive patients suggest that it is acceptable providing no other bacterial or viral illnesses are active.

The postulated mechanism for its effectiveness is compression of the thecal space and elevating the subarachnoid pressure. Maintenance of the therapeutic effect is attributed to clot preventing further CSF leaks.(26) Blood in the epidural space will spread between 7 and 14 spinal segments. The mean spread of blood is six segments upward and three segments downward.(27)

The timing of the EBP is debated. Loeser noted a 71\% failure rate if the epidural blood patch was done within 24 hours of dural puncture as compared to a $4 \%$ failure rate if done greater than 24 hours. Subsequent studies have also noted this finding. ${ }^{(28)}$

Outcome: The technique has a success rate of $70-98 \%$ if carried out more than $24 \mathrm{~h}$ after the dural puncture.(29) If an epidural blood patch fails to resolve the headache, repeating the blood patch has a similar success rate. Failure of the second patch and repeating the patch for a third or fourth time has been reported. However, in the presence of persistent severe headache, an alternative cause should be considered. $(18,19,20)$

Complications: Immediate exacerbation of symptoms and radicular pain have been described. These symptoms do not persist and resolve with the administration of simple painkillers. Long term complications of epidural blood patch are rare. A single case report of an inadvertent subdural epidural blood patch described non - postural, persistent headache and lower extremity discomfort.

Epidural saline: Concerns have been expressed about the potential danger of an autologous epidural blood patch for the treatment of post - dural puncture headache. The immediate resolution of the headache with a blood patch is attributable to thecal compression raising the CSF pressure. An 
epidural injection of saline( ${ }^{(30)}$ would, in theory, produce the same mass effect, and restore normal CSF dynamics.

As saline is a relatively inert and sterile solution, epidural saline bolus or infusion appears to be an attractive alternative. Regimens that have been advocated include: (i) 1.0-1.5 litre of epidural Hartmann's solution over 24h, starting on the first day after dural puncture; (ii) up to $35 \mathrm{ml} / \mathrm{hr}$ of epidural saline or Hartmann's solution for $24-48 \mathrm{~h}$, or after development of the headache; (iii) a single $30 \mathrm{ml}$ bolus of epidural saline after development of headache and (iv) 10-120 ml of saline injected as a bolus via the caudal epidural space.

Epidural, intrathecal and parenteral opiodis: A number of authors have advocated the use of epidural,(31) intrathecal(32) or parenteral morphine;(33) the majority of these reports are either case reports or inadequately controlled trials. Some of the studies used epidural morphine after the onset of headache; others used epidural or intrathecal morphine as prophylaxis or in combination with an intrathecal catheter. A controlled trial of intrathecal fentanyl as prophylaxis found no evidence of a reduction in the incidence of post - spinal headache after dural puncture with a 25- gauge spinal needle.

Intrathecal Catheters: After accidental dural perforation with a Tuohy needle, it has been suggested that the placement of a spinal catheter through the perforation may provoke an inflammatory reaction that will seal the hole. This data supports the placement of a subarachnoid catheter after the occurrence of a wet tap and leaving it in for 24 hours. Extreme care should be used when a subarachnoid catheter is left in place for 24 hours. A case report discussed a patient who had the catheter adapter dislodge, resulting in a CSF leak while another presented a patient who developed meningitis. $(34,35)$

CONCLUSION: PDPHA continues to be a problem following neuraxial anesthesia. It is due to a decrease in CSF volume intracranially. It is not easily prevented, with an intrathecal catheter holding the most promise. It is easily treated with an EBP. An EBP performed within the first 24 hours of dural puncture may decrease its effectiveness. After 24 hours, an EBP should be done in the symptomatic patient.

\section{REFERENCES:}

1. Harrington B. E. "Postdural puncture headache and the development of the epidural blood patch," Regional Anesthesia and Pain Medicine, 2004; 29; 136-163.

2. Turnbull D. K. and Shepherd D. B. "Post-dural puncture headache: pathogenesis, prevention and treatment," British Journal of Anaesthesia, 2003; 91; 718-729.

3. Norris M. C., Leighton B. L., and DeSimone C. A."Needle bevel direction and headache after inadvertent dural puncture," Anesthesiology, 1989; 70; 729-731.

4. Flaatten, Thorsen T, Askeland B, et al. Puncture technique and postural postdural puncture headache. A randomized, double - blind study comparing transverse and parallel puncture. Acta Anaesthsiol Scand 1998; 42: 1209-14.

5. Richman JM, Joe EM, Cohen SR. et al. Bevel direction and postdural puncture headache: a metaanalysis. The Neurologist 2006; 12: 224-228. 


\section{REVIEW ARTICLE}

6. Reina M. A., M. Dittmann, A. L. Garcia, and A. Van Zundert, "New perspectives in the microscopic structure of human dura mater in the dorsolumbar region," Regional Anesthesia, vol. 22, no. 2, pp. 161-166, 1997.

7. Levine Dn, Rapalin 0. the pathophysiology of lumbar puncture headache. J Neurol Science 2001; 192: 1-8.

8. Pannullo SC, Reich JB, Krol G, Deck MDF, Posner JB. MRI Changes in intracranial hypotension. Neurology 1993; 43: 919-926.

9. Grant R, Condon B, Hart I, Teasdale GM. Changes in intracranial CSF volume after lumbar puncture and their relationship to post-LP headache. J Neurol Neurosurg Psychiatry 1991; 54: $440-2$.

10. $\mathrm{Wu} \mathrm{Cl}$, Rowlingson $\mathrm{Aj}$, Cohen $\mathrm{SR}$, et al. gender and post - dural puncture headache. Anesthesiology 2006; 105: 613-8.

11. Angle PJ, Kronberg JE, Thompson DE, et al. Dural tissue trauma and cerebrospinal fluid leak after epidural needle puncture: Effect of needle design, angle, and bevel orientation. Anesthesiology 2003; 99: 1376-82.

12. Lybecker H, Moeller JT, may 0, et al. Incidence and prediction of postdural puncture headache. A prospective study of 1021 spinal anesthesias. Anesth Analg 1990; 70: 389-94.

13. Janssens E, Aerssens $P$, Alliet $P$, et al. post - dural puncture headaches in children. A literature review. Eur J Pediatr 2003; 162: 117-21.

14. Evans RW, Armon C, Frohman Em, et al. Assessment/Prevention of post-lumbar puncture headaches. Report of the Therapeutics an technology Assessment Sub-committee of the Academy of Neurology. Neurology 200; 55: 909-14.

15. Wang LP Schmidt JF. Central nervous side effects after lumbar puncture. Dan Med Bull 199; 44: 79-81.

16. Arcand G, Girard F, Mc Cormack M, et al. Bilateral sixth cranial nerve palsy after unintentional dural puncture. Can J Anesth 2004;51:821-3

17. Bechard P, Perron G, Larochelle D, et al. Epidural blood patch in the treatment of abducens palsy after a dural puncture. Can J Anaesth 2007;54:146-50

18. T.Horlocker, "Complications of spinal and epidural anesthesia," Anesthesiology Clinics of North America, vol. 18, no. 2, pp. 461-485, 2000.

19. Abram S. E., "Treatment of lumbosacral radiculopathy with epidural steroids," Anesthesiology, vol. 91, no. 6, pp. 1937-1941, 1999.

20. Errando C. L., J. C. Rowlingson, and P. S. Hodgson, "Transient neurologic syndrome, transient radicular irritation, or postspinal musculoskeletal symptoms: are we describing the same "syndrome"in all patients?" Regional Anesthesia and Pain Medicine. 2001; 26; 178-180.

21. Thornberry EA, Thomas TA. Posture and post-spinal headache. A controlled trail in 80 obstetric patients. Br J Anaesth 1988; 60: 195-7.

22. Cook PT, Davies MJ. Bed rest and postlumbar puncture headache. The effectiveness of 24 hours recumbency in reducing the incidence of postlumbar puncture headache. Anaesthesia 1989; 44: 389-91.

23. Sechzer PH, Abel L. Post-spinal anesthesia headache treated with caffeine. Evaluation with demand method. Part 1. Current Therapeutic Research 1978; 24: 307-12. 
24. Ghaleb A, Khorasani A, Mangar D.Int J Gen Med. 2012; 5: 45-51. doi: 10.2147/IJGM.S17834. Epub 2012 Jan 12.

25. Gormely JB. Treatment of Postspinal headache, Anesthesiology 1960: 21: 565-6.

26. Rosenberg PH, Heavner JE. In vitro study of the effect of epidural blood patch on leakage through a dural puncture. Anesth Analg 1985; 64:501-4.

27. Szeinfeld M, Ihmeidan IH, Moser MM, et al.Epidural blood patch: Evaluation of the volume and spread of blood injected into the epidural space. Anesthesiology 1986; 64: 820-2.

28. Loeser EA, Hill GE, et al. Time vs.Success rate for epiduralk blood poatch. Anesthesiology 1978; 49: 147-8.

29. Abouleish E, Vega S, Blendinger I, Tio TO. Long-term follow-up of epidural blood patch. Anesth Analg 1975; 54: 459-63.

30. Liu SK, Chen KB, Wu RS, Lin BC, Chang CS, Liu YC, Hung CJ. Acta Anaesthesiol Taiwan. 2006 Dec; 44 (4): 227-30.

31. Eldor J, Guedj P, Cotev S. Epidural morphine injections for the treatment of postspinal headache. Can J Anaesth 1990; 37: 710-11.

32. Cohen S, Amar D, Pantuck EJ, Singer N, Divon M. Decreased incidence of headache after accidental dural puncture in caesarean delivery patients receiving continuous postoperative intrathecal analgesia. Acta Anaesthesiol Scand 1994; 38: 716-18.

33. ldor J. Opiate treatment of post-dural puncture headache. Acta Anaesthesiol Scand 1995; 39: 1140.

34. Cohen S, Stricker P, Sakr A. Cerebrospinal fluid leak after disconnection of an intrathecal catheter adapter placed after accidental dural puncture. Reg Anesth Pain med 2005; 30: 591.

35. Cohen S, Hunter CW, Sakr A, Hijazi RH. Meningitis following intrathecal catheter placement after accidental dural puncture. Int J Obstet Anesthe 2006; 15: 172.

\section{AUTHORS:}

1. Prabhavathi R.

2. Chaitanya Kumar G.

3. Narasimha Reddy P.

\section{PARTICULARS OF CONTRIBUTORS:}

1. Associate Professor, Department of Anaesthesia, Narayana Medical College Hospital.

2. Assistant Professor, Department of Anaesthesia, Narayana Medical College Hospital.

3. Professor and HOD, Department of Anaesthesia, Narayana Medical College Hospital.

\author{
NAME ADDRESS EMAIL ID OF THE \\ CORRESPONDING AUTHOR: \\ Dr. Prabhavathi Ravipathi, \\ Associate Professor, \\ Department of Anesthesia, \\ Narayana Medical College, \\ Chintareddy Palem, \\ Nellore-524002. \\ Email: prabhavathi95gmc@gmail.com
}

Date of Submission: 06/08/2014.

Date of Peer Review: 07/08/2014.

Date of Acceptance: 12/08/2014.

Date of Publishing: 16/08/2014. 